\title{
LOCALIZED OPTICAL STATES IN A DEFECT-CONTAINING LIQUID-CRYSTAL STRUCTURE ADJACENT TO THE METAL
}

\author{
Maxim V. Pyatnov ${ }^{1,}{ }^{*}$, Stepan Ya. Vetrov ${ }^{1,2}$, IVAn V. Timofeev 2,3 \\ ${ }^{1}$ Institute of Engineering Physics and Radio Electronics, Siberian Federal University, Krasnoyarsk, Russia \\ ${ }^{2}$ Kirensky Institute of Physics, Federal Research Center KSC SB RAS, Krasnoyarsk, Russia; \\ ${ }^{3}$ Laboratory of Nonlinear Optics and Spectroscopy, Siberian Federal University, Krasnoyarsk, Russia \\ *Corresponding author: MaksPyatnov@yandex.ru
}

Received XX Month XXXX; revised XX Month, XXXX; accepted XX Month XXXX; posted XX Month XXXX (Doc. ID XXXXX); published XX Month XXXX

\begin{abstract}
The possibility of existence of the localized optical states with the maximum field intensity at the interface between the metal and cholesteric liquid crystal in a defect-containing cholesteric liquid crystal is theoretically grounded. It is established that the localization occurs at almost any defect thicknesses rather than only at the half-wave thickness, at which the loss of the polarization dependence of diffraction reflection and the absence of field localization are observed for the structure without the metallic layer. It is demonstrated that the spectral properties can be controlled by external fields affecting the liquid crystal. At large thicknesses of the cholesteric liquid crystal between the defect and metallic layer, the spectrum contains the pronounced peaks corresponding to the edge modes. The results obtained open new opportunities for controlling the transmittance spectrum, polarization and localization of light in optoelectronic elements based on the investigated system. (c) 2017 Optical Society of America
\end{abstract}

OCIS codes: (240.6680) Surface plasmons; (160.3710) Liquid crystals; (230.3720) Liquid-crystal devices; (230.5298) Photonic crystals.

http://dx.doi.org/10.1364/JOSAB.99.099999

\section{INTRODUCTION}

Special localized modes called the surface states can exist between different media. The surface states in photonic-crystal (PC) media are promising objects for fundamental research and application [1]. The optical Tamm state (OTS) is one of the intensively investigated surface electromagnetic waves [2]. This state is an electromagnetic analog of bound electronic states on the crystal surface in physics of condensed matter. The OTS can be excited at the normal incidence of light between two different PCs with overlapping band gaps [2] or between a PC and a medium with the negative permittivity $[3,4]$. Recently, the OTS has been obtained at the interface between a one-dimensional PC and organic polymer layer doped with a dye [5]. The OTS excited at the interface with a metal is called also the Tamm plasmon polariton. In contrast to the surface plasmon polaritons, the OTS can be formed by both the TE and TM linear polarizations and excited at any angles of light incidence. The OTS manifests itself in experiments as a narrow resonance in the transmission or reflection spectrum of a sample. In recent years, the hybrid states representing the interactions between the OTS and resonances of different nature have been intensively studied [6-10]. The OTS and hybrid states based on them are applied in lasers [11-13], sensors $[14,15]$, photovoltaic elements [16], top-emitting white organic light-emitting devices [17], and bistable switches [18].

Cholesteric liquid crystals (CLCs) represent a class of one-dimensional self-assembling chiral PCs $[19,20]$. They are formed by elongated molecules arranged in helical structures. On average, these molecules are oriented similarly in the plane perpendicular to the helix axis. Their orientation direction is characterized by a director. The director continuously rotates along the axis with the formation of a birefringent structure. At the normal incidence of light in the wavelength range of $p n_{o}<\lambda<p n_{e}$, where $p$ is the helix pitch and $n_{o}$ and $n_{e}$ are the ordinary and extraordinary refractive indices, there is a CLC photonic bad gap for the circular polarization with a handedness sign identical to that of the helix. This circularly polarized radiation is reflected from the CLC with a reflectance depending on the layer thickness. There is the only selective reflection band in the spectrum for the light propagating along the helix axis. The radiation with the circular polarization different from the CLC helix handedness does not experience the diffraction reflection.

In contrast to other types of one-dimensional PCs, for the CLC there exists the exact solution of the Maxwell equations at the normal incidence of light [19]. The eigensolutions are the superposition of two circularly polarized plane waves. These can be either two waves with the opposite circular polarizations propagating in one direction or two waves with the same circular polarization propagating in the opposite directions.

The advantage of CLCs is their tunability. The variations in temperature or external electric or magnetic fields and photoorientation effect can change the CLC structure [20], e.g., increase or decrease the cholesteric helix pitch and thereby change the band gap position. Another important feature of the CLCs is that it can have two or even three optically stable states. These remarkable properties allow the CLCs to be used in various optical devices $[21,22]$.

The attempts to localize light at the interface between the CLC and metallic layer were made in [23-25]. We showed that this problem is 
nontrivial due to the polarization features of light reflection from the CLC and metal [24]. The light reflected from the CLC retains its circular polarization, but the metal changes it. The polarization change can be compensated using an additional anisotropic layer. The role of such a layer was played by a quarter-wave plate inserted between the CLC and metal [23] and by an oppositely handed CLC [25]. One more way to find the OTS at the CLC boundary was the use of a chirality-preserving mirror instead of the metal $[26,27]$.

In this study, we propose a new method for localizing the light between the CLC and metallic film by using a defect layer in the cholesteric.

\section{DESCRIPTION OF THE SYSTEM}

Some authors [28-32] studied the systems in the form of a CLC with the anisotropic defect layer. Alaverdyan et al. [32] developed optical CLC cells with electrodes located perpendicular to the CLC helix axis. Using these cells, they manage to untwist the CLC helix in the middle of the layer and thereby create a planar defect. Hsiao et al. [29] applied the thermodielectric effect to form a local strain in the middle of a one-dimensional periodic helical structure.

Based on these studies, we investigated the structure consisting of a metallic film and a CLC with the anisotropic planar defect (Fig. 1). The CLC parameters used were the extraordinary and ordinary refractive indices of $n_{e}=1.71$ and $n_{o}=1.54$, respectively, and a helix pitch of $p=0.4$ $\mu \mathrm{m}$. These parameters are typical of the mixture of a Merck S-811 chiral center and a Merck E44 nematic liquid crystal [33]. In this case, the CLC band gap lies between 616 and $684 \mathrm{~nm}$. In the middle of the CLC layer, there is a planar anisotropic defect with thickness $d$ with the ordinary and extraordinary refractive indices equal to the CLC refractive indices. This is analogous to the case of the CLC helix untwisted on length $d$. The crystal is assumed to be right-handed. The CLC thickness on the left and on the right of the defect is $2 \mu \mathrm{m}$. In the simulation, we did not take into account the angle of a possible twist defect between the director at the first CLC output and initial director of the second CLC. Such a defect will not qualitatively change the results of this work. To ignore the twist defect, below the CLC thickness is only changed by an integer number of periods. The structure is surrounded by a medium with the refractive index equal to the averaged CLC refractive index $n_{\text {ext }}=\left(n_{e}+n_{0}\right) / 2$. The metallic film thickness was $d_{m}=50 \mathrm{~nm}$ and the film permittivity was specified in the form of the Drude approximation

$$
\varepsilon(\omega)=\varepsilon_{0}-\frac{\omega^{2}}{\omega(\omega+i \gamma)},
$$

where $\varepsilon_{0}=5$ is the ionic core contribution, $\hbar \omega_{p}=9 \mathrm{eV}$ is the plasma frequency, and $\gamma=0.02$ is the reciprocal relaxation time [34]. These parameters are typical of silver. The transmission spectra and field intensity distribution in the structure were studied using the matrix method for calculating the layered anisotropic Berreman structures [35].

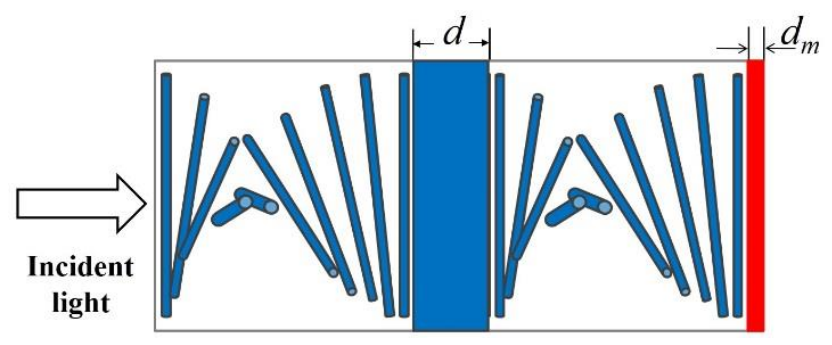

Fig. 1. Schematic of the investigated structure

\section{HALF-WAVE DEFECT}

As was shown in $[28,31]$, the presence of a half-wave defect layer in the CLC structure leads to the loss of the polarization dependence of diffraction reflection. The CLC starts reflecting light of any polarization rather than only the radiation circularly polarized in the direction coinciding with the cholesteric helix handedness. When such a defect CLC is combined with a metal, transmission peaks can arise in the transmission spectrum at the frequencies corresponding to the Bragg reflection zone. Each spectral peak corresponds to the localized state. In the specified CLC, the zone center is $\lambda_{0}=650 \mathrm{~nm}$. At this wavelength, the halfwave defect thickness is $d=\lambda_{o} / 2\left(n_{e}-n_{o}\right)=1.91 \mu \mathrm{m}$.

Figure 2 shows the electric field intensity distribution for the righthand circular polarization at a wavelength of $645.4 \mathrm{~nm}$. The $|\mathrm{E}|^{2}(\mathrm{z}) \mathrm{de}-$ pendence for the rest peaks (inset in Fig. 2) is analogous. It can be seen that the larger part of the field is localized at the CLC/metal interface. The localized mode field decay inside the metal is related to the negative permittivity of the metallic film; the decay inside the CLC is caused by the Bragg reflection. A part of the field is localized on the defect with the maximum at the half-wave layer center. The spectral peaks arise at any polarization of the incident radiation; however, the transmittances are different.

The occurrence of light localization for the diffracting (right-hand circular R) and nondiffracting (left-hand circular L) polarizations is illustrated in Fig. 3. If the light with the right-hand diffracting polarization falls onto the CLC layer (Fig. 3a), then a part of the light transmitted through this layer approximately preserves its polarization at the CLC output. After transmitting through the half-wave defect, the light will become L-polarized. The L polarization will be preserved after passing through the second CLC layer. Upon reflection from the metal, the L polarization will turn to the R polarization. Upon reflection from the CLC, the $\mathrm{R}$ polarization is retained. Upon repeated reflection from the metal, the R polarization transforms to the L polarization and the light propagates through the crystal in the opposite direction. After passing through the half-wave defect, the light will become R-polarized and reflect from the CLC. After repeated passing through the half-wave defect, the light will become L-polarized again. The procedure is repeated.

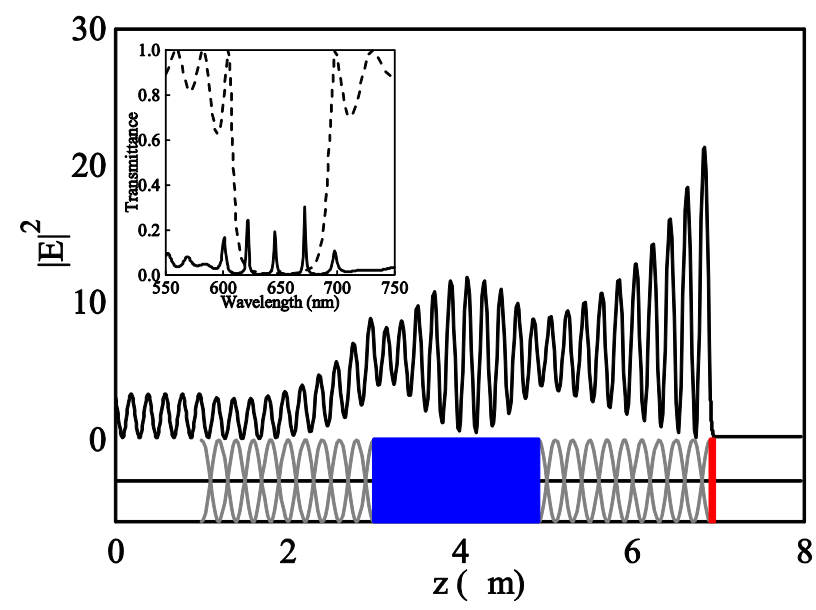

Fig. 2. Spatial local field intensity distribution in the structure CLC-halfwave defect- CLC-metal. The refractive indices of the CLC and the defect: $n_{e}=1.71, n_{o}=1.54$, CLC helix pitch $\mathrm{p}=400 \mathrm{~nm}$. CLC contains 10 periods on the left and on right of the defect. The thickness of the defect is $d=1.91 \mu \mathrm{m}$, the thickness of the silver film is $d_{m}=50 \mathrm{~nm}$. For the environment $n_{\text {ext }}=1.625$. The intensity is normalized to the input intensity at $\lambda=645.4 \mathrm{~nm}$. Inset: transmission spectra of twenty CLC periods (dashed line) and the entire structure (solid line) at the normal incidence of light with the right-hand circular polarization. 

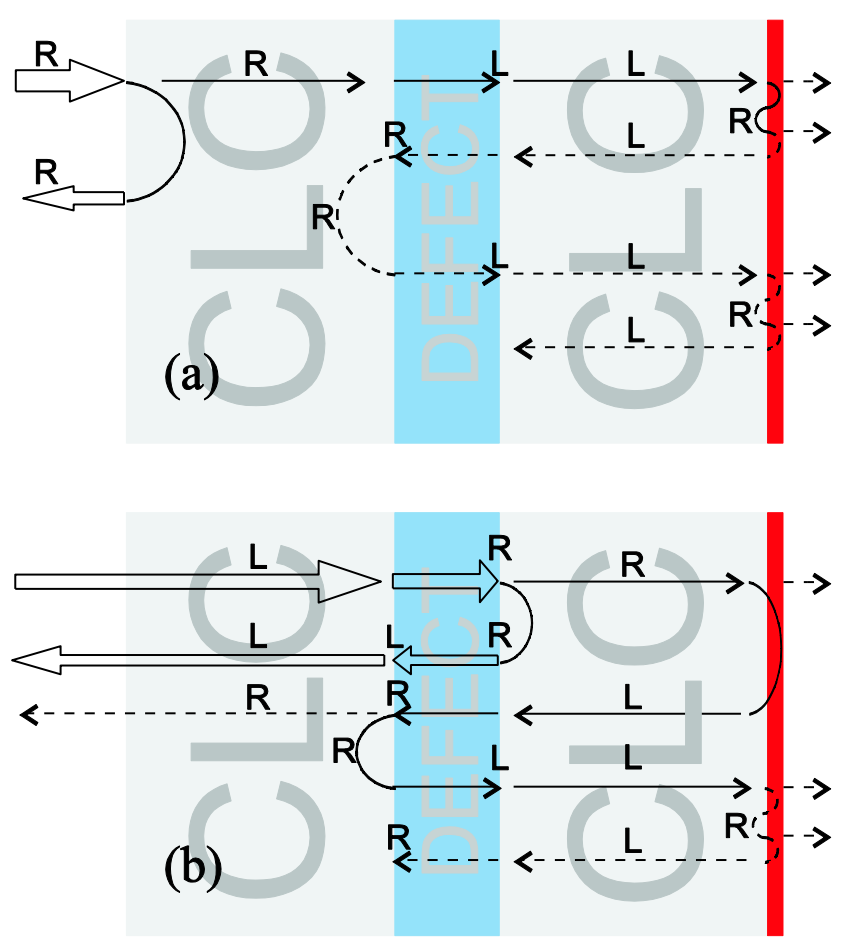

Fig. 3. Mechanisms of light localization in the structure CLC-defectCLC-metal with a half-wave defect for (a) the right-handed circular polarization $R$ and (b) left-handed circular polarization $L$ of the incident radiation.

The situation is similar for the nondiffracting left-handed polarization L (Fig. 3b). The incident light passes freely through the CLC. After passing through the half-wave defect, the light will become R-polarized. A part of the radiation will be reflected from the CLC and, when passing through the defect and first CLC layer, will leave the structure. A smaller part of the light passed through the second CLC layer and reflected from the metal will become L-polarized. The situation will become analogous to the previous one.

\section{DEFECT WITH AN ARBITRARY THICKNESS}

The CLC containing the anisotropic half-wave defect reflects light of any polarization at the Bragg frequencies. If the anisotropic layer thickness is different from half-wave, this effect is not observed. Then, similarly to the case of the isotropic defect in the CLC band gap, the transmission peaks corresponding to the localized modes arise for diffracting light polarization and dips, for the nondiffracting one [36]. For the linear polarizations, the distorted band gap occurs.

One should expect that the optical states localized at the interface between the metal and CLC with such a defect do not occur. Nevertheless, the calculation shows that at the anisotropic layer thickness different from half-wave, the localized states occur. The transmission peaks start arising in the spectrum at almost any defect layer thickness (Fig. 4). When the defect thicknesses are small, addition of the metallic layer does not qualitatively change the transmission spectrum. When a metal is added to the defect-containing CLC, absorption of the system will increase and the transmittances at any incident light polarization will decrease. The similar situation is observed for the isotropic defect layer of an arbitrary thickness in the CLC. For comparison, Fig. 5a shows the transmission spectra of the CLC with the isotropic defect and structure CLC-isotropic defect-CLC-metal.

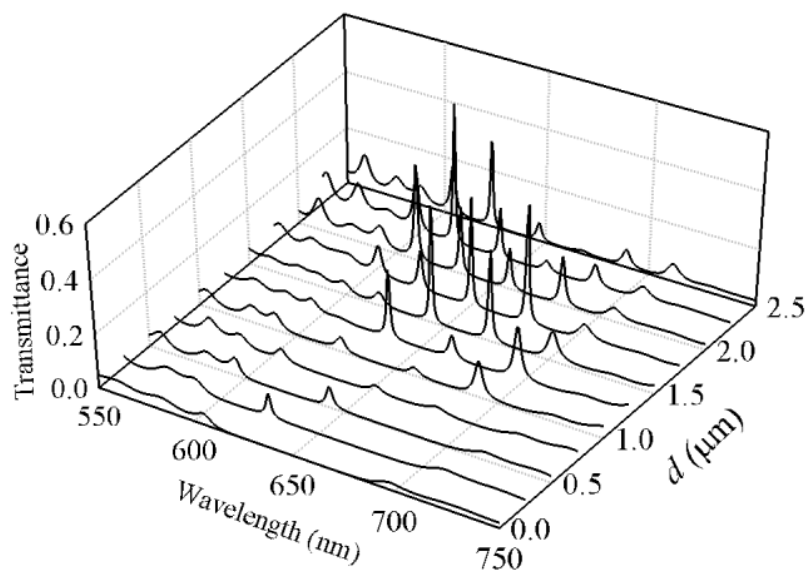

Fig. 4. Transmission spectrum of the structure CLC-defect-CLC-metal for different defect layer thicknesses $d$. The rest parameters are as in Fig. 2.

When the isotropic defect layer is changed for the anisotropic one with the same thickness, the transmission spectrum qualitatively
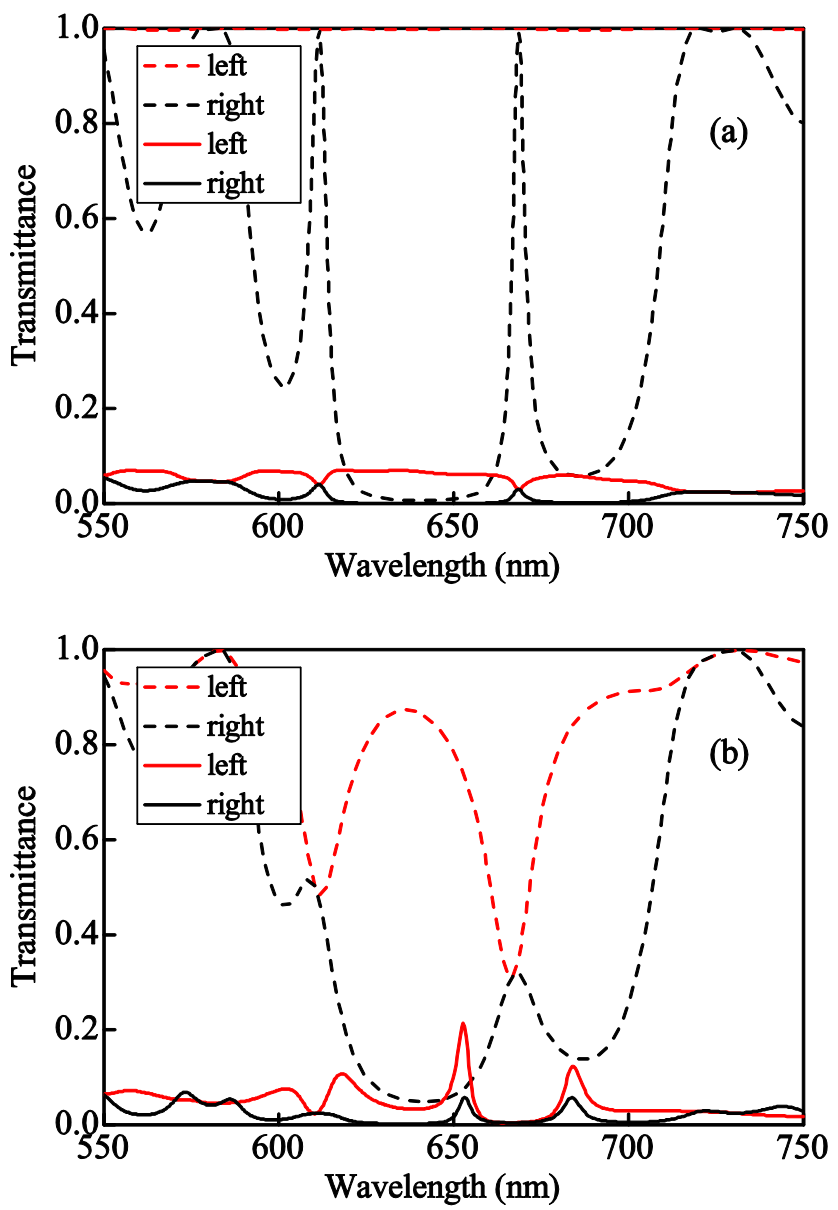

Fig. 5. Transmittance spectrum of the CLC containing a defect with a thickness of $d=0.96 \mu \mathrm{m}$ (dashed line) and CLC-defect-CLC-metal structure (solid line) for the circular polarizations: (a) the CLC contains the isotropic defect and (b) the CLC contains the anisotropic defect. The rest parameters are as in Fig. 2. 
changes (Fig. 5b). At the frequencies lying inside the band gap and different from the frequencies of CLC defect mode peaks, two resonances form, each corresponding to the localized state and the distribution of the squared electric field strength is analogous to that shown in Fig. 2. At the larger thickness $d$, the number of peaks increases. As the anisotropic defect layer thickness grows, the role of this layer in the polarization variation becomes more important. The spectrum significantly changes. The half-wave layer transforms the circular polarizations to one another. The anisotropic layer of different thickness transforms the circular polarization to elliptic or linear (at the quarter-wave layer). The CLC only reflects the circular component coinciding with its helix handedness. The thin anisotropic layer weakly transforms the polarization; therefore, the thin defect does not lead to the occurrence of localized states.

\section{CONTROLLING THE SPECTRAL PROPERTIES}

An important property of the CLC is its high sensitivity to external factors. The electric, magnetic, thermal, mechanical, and other effects can change the crystal structure. For example, the helix pitch can be changed, which opens the opportunity for controlling the transmission spectrum. We studied the effect of the helix pitch on the transmission spectrum of the CLC-defect-CLC-metal structure. The change in the pitch of the entire cholesteric leads to the peak shift in accordance with the Bragg condition. In addition, the helix pitch can be changed not only
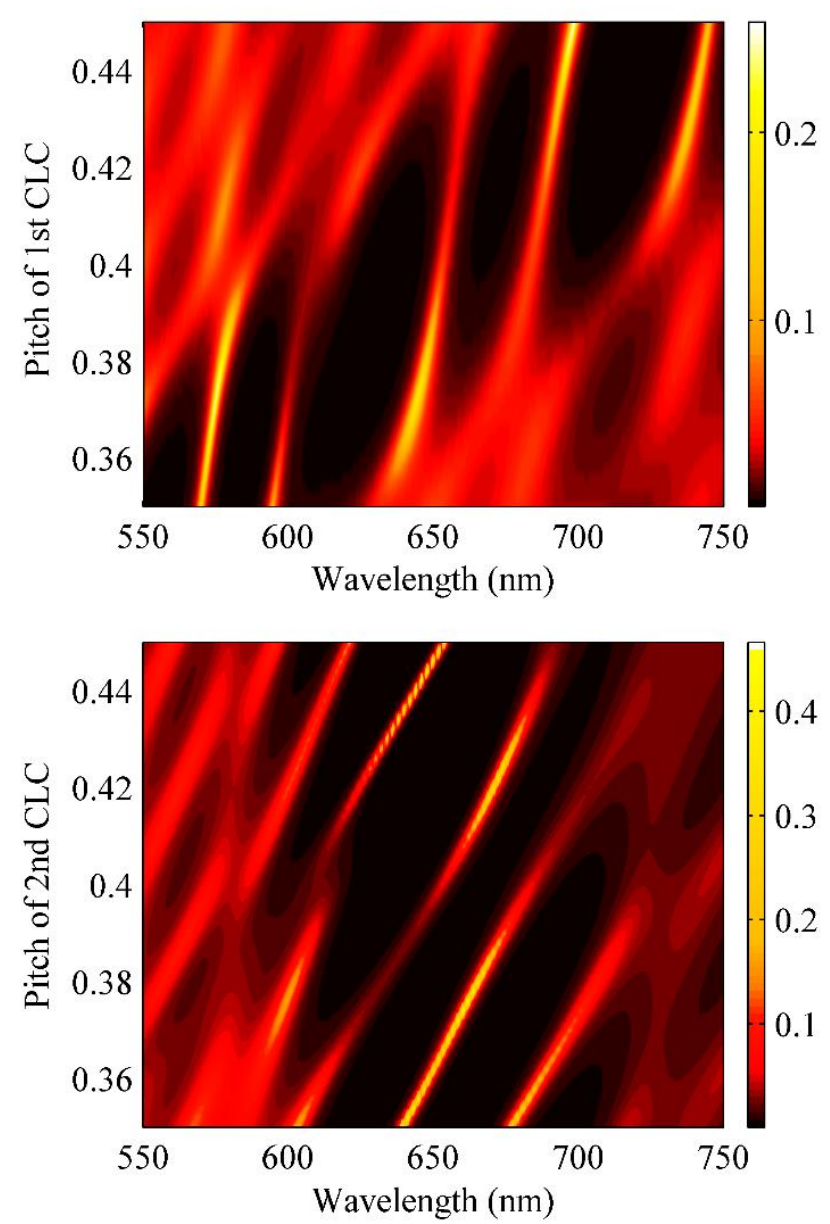

Fig. 6. Transmission spectrum of the CLC-defect-CLC-metal structure for the right-handed circular polarization of incident light at different cholesteric helix pitches (a) on the left and (b) on the right from the defect. The rest parameters are as in Fig. 2. in the entire CLC, but also in its left (Fig. 6a) or right (Fig. 6b) part with respect to the defect. It was found that the change in the helix pitch of the helix pitch of the second CLC. Figure 6a shows that the increase in the CLC pitch on the left from the defect leads to the peak shift to the longwavelength region in accordance with the Bragg condition. When the CLC pitch changes on the right from the defect, the peaks shift insignificantly.

The investigated system can be considered as a composition of two resonators. The first resonator mirrors are the CLC layers on the left and on the right from the defect layer. The metallic layer and combination of the first CLC and phase-changing anisotropic layer can be considered as mirrors of the second resonator containing the second CLC layer. However, these resonators are very special. Separately, the resonator is only the CLC layer containing the defect layer, while the CLC-metal or defect-CLC-metal system is not a resonator. The CLC with the structural defect is a part of the larger resonator.

Varying the helix pitch of the second CLC layer, we change the optical thickness of the medium inside the second resonator, whereas varying the helix pitch of the first CLC layer, we change the position of the Bragg reflection zone of the mirror formed by the combination of the first CLC and defect layer. Therefore, the peak shift in Fig. $6 \mathrm{~b}$ is much weaker than in the case illustrated in Fig. 6 a.

The transmission spectrum depends significantly on the defect position in the CLC. Let us consider the change in the transmission spectrum of the structure at different numbers of periods in the second CLC. Assume the CLC helix pitches on the left and on the right from the defect to coincide and be equal to $400 \mathrm{~nm}$ and the defect thickness to correspond to a quarter-wave layer $(d=0.96 \mu \mathrm{m})$. The calculated spectrum (Fig. 7) shows that with increasing number of periods the number of peaks in the spectrum grows. The dashed line shows the band gap boundaries in the ideal thick CLC, which lies between 616 and $684 \mathrm{~nm}$ at the parameters used in the simulation. Note the occurrence of peaks beyond these wavelengths. Figure 8 shows the distribution of the squared absolute value of the electric field strength at wavelengths of 683,695 , and 710 $\mathrm{nm}$ for 40 periods of the second CLC (the layer thickness is $8 \mu \mathrm{m}$ ). This field localization type allows us to conclude that these peaks correspond to the edge modes of the first-, second-, and third-order CLCs [37]. In addition, it can be seen that the field is relatively weakly localized on the defectlayer and CLC/metal interface. The analogous peaks are observed in Fig. $6 \mathrm{~b}$ and inset in Fig. 2, but for ten periods of the second CLC the field is localized much stronger on the defect and CLC/metal interface.

The increase in the number of periods of the first CLC layer does not qualitatively change in the transmission spectrum. In this case, the

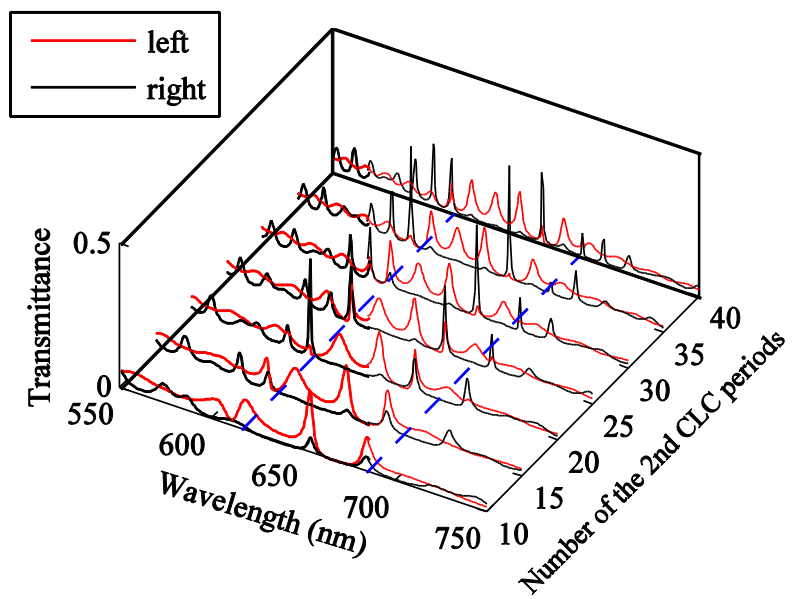

Fig. 7. Transmission spectrum of the CLC-defect-CLC-metal structure. The rest parameters are as in Fig. 2 . The dashed lines show the edges of the CLC band gap. 


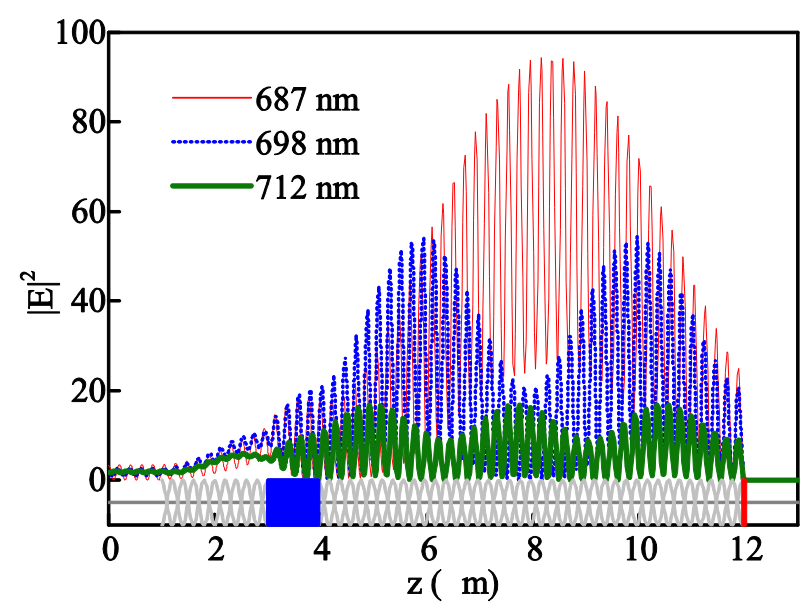

Fig. 8. Spatial distribution of the local electric field intensity in the structure with a defect with a thickness of $d=0.96 \mu \mathrm{m}$ and 40 CLC periods on the right from the defect for the right-handed circular polarization. The CLC on the left has ten periods.

transmittance of the resonator mirror formed by the metal and combination of the first CLC layer and defect grows, but the optical thickness of the cavity between them does not change. The right-handed circular polarization is reflected almost fully by the first mirror. Therefore, its peaks are not seen. For the left-handed polarization, the peaks almost do not shift.

The CLC tunability makes the investigated structure useful for various optical devices, such as sensors and solar cells [38]. In this case, the light is localized in different geometrical parts of the structure at different frequencies.

\section{CONCLUSIONS}

Using the numerical simulation, we demonstrated the existence of the optical electromagnetic states localized in the structure containing a silver film and a cholesteric liquid crystal with the planar anisotropic defect. This defect can be either the layer of a foreign material embedded in the CLC or an untwisted part of its helix. The helix can be untwisted, e.g., by applying an external electric field perpendicular to CLC helix layer. When the CLC structure contains such a defect, the light can be partially localized at the CLC/metal interface. The established states manifest themselves as peaks in the transmission spectrum of the structure.

It was established that the presence of a defect of almost any thickness rather than only half-wave one leads to the light localization at the $\mathrm{CLC} /$ metal interface. The limitation is imposed only on its thickness. The thin anisotropic layer cannot significantly change the radiation polarization, which is necessary to localize the light at the CLC/metal interface. The necessity in such a change is related to the different polarization properties of the system elements.

We showed the possibility of controlling the transmission spectrum via changing the CLC helix pitch in applied fields. It was established that the variation in the CLC helix pitch on the left from the defect changes the spectrum much stronger than the variation on the right from it. This is related to the resonator properties of the structure under study. The investigated system can be considered as a composition of two resonators. The first resonator mirrors are the CLC layers on the left and on the right from the defect layer. The metallic layer and combination of the first CLC layer and defect can be considered to be the second resonator mirrors. Basing on these considerations, the predicted phenomena were grounded.
Adding an optically active material to the second CLC or defect layer can allow laser generation in the system under study. The high sensitivity to external fields will ensure the tunability of the emission spectrum of the sample.

Funding. Russian Foundation for Basic Research and Government of Krasnoyarsk Territory, Krasnoyarsk Region Science and Technology (17-42-240464).

\section{REFERENCES}

1. A. P. Vinogradov, A. V. Dorofeenko, A. M. Merzlikin, A. A. Lisyansky, "Surface states in photonic crystals," Phys. Usp. 53, 243-256 (2010).

2. A. V. Kavokin, I. A. Shelykh, G. Malpuech, "Lossless interface modes at the boundary between two periodic dielectric structures," Phys. Rev. B 72, 233102 (2005).

3. M. Kaliteevski, I. Iorsh, S. Brand, R. A. Abram, J. M. Chamberlain, A. V. Kavokin, and I. A. Shelykh, "Tamm plasmon-polaritons: possible electro-magnetic states at the interface of a metal and a dielectric Bragg mirror," Phys. Rev. B 76, 165415 (2007).

4. S. Ya. Vetrov, R. G. Bikbaev, I. V. Timofeev, "The optical Tamm states at the edges of a photonic crystal bounded by one or two layers of a strongly anisotropic nanocomposite," Opt. Comm. 395, C. 275-281 (2017).

5. S. Núñez-Sánchez, M. Lopez-Garcia, M. M. Murshidy, A. G. AbdelHady, M. Serry, A. M. Adawi, J. G. Rarity, R. Oulton and W. L. Barnes, "Excitonic Optical Tamm States: A Step toward a Full Molecular-Dielectric Photonic Integration," ACS Photon. 3, 743-748 (2016).

6. B. I. Afinogenov, V. O. Bessonov, A. A. Nikulin, and A. A. Fedyanin, "Observation of hybrid state of Tamm and surface plasmon-polaritons in one-dimensional photonic crystals," Appl. Phys. Lett. 103, 061112 (2013).

7. M. Kaliteevski, S. Brand, R. A. Abram, I. Iorsh, A. V. Kavokin, and I. A. Shelykh, "Hybrid states of Tamm plasmons and exciton polaritons," Appl. Phys. Lett. 95, 251108 (2009).

8. R. Brückner, M. Sudzius, S. I. Hintschich, H. Frob, V. G. Lyssenko, and K. Leo, "Hybrid optical Tamm states in a planar dielectric microcavity," Phys. Rev. B 83, 033405 (2011).

9. Rahman Sk. S.-U., T. Klein, S. Klembt, J. Gutowski, D. Hommel, anf K.Sebald, "Observation of a hybrid state of Tamm plasmons and microcavity exciton polaritons," Sci. Rep. 6,34392 (2016).

10. H. Liu, J. Gao, Z. Liu, X. Wang, H. Yang, and H. Chen, "Large electromagnetic field enhancement achieved through coupling localized surface plasmons to hybrid Tamm plasmons," JOSA B 32, 20612067 (2015).

11. C. Symonds, G. Lheureux, J. P. Hugonin, J. J. Greffet, J. Laverdant, G. Brucoli, A. Lemaitre, P. Senellart, and J. Bellessa, "Confined Tamm plasmon lasers," Nano Lett. 13, 3179-3184 (2013).

12. G. Lheureux, S. Azzini, C. Symonds, P. Senellart, A. Lemaître, C. Sauvan, and J. Bellessa, "Polarization-controlled confined Tamm plasmon lasers, " ACS photon., 2, 842-848 (2015).

13.Z. Zhang, Y. Li, S. Wang, H. Lu, Y. Sun, H. Jiang, and H. Chen, "Metasurface-enhanced optical Tamm states and related lasing effect," J. Opt. Soc. Am. B 32, 1624-1629 (2015).

14.S.-G. Huang, K.-Ping Chen, and S.-C. Jeng, "Phase sensitive sensor on Tamm plasmon devices," Opt. Mater. Exp. 7, 1267-1273 (2017).

15. B. Auguié, M. C. Fuertes, P. C. Angelomé, N. L. Abdala, G. J. A. A. Soler Illia, and A. Fainstein, "Tamm plasmon resonance in mesoporous multilayers: toward a sensing application," ACS Photon. 1, 775-780 (2014).

16. X.-L. Zhang, J.-F. Song, X.-B. Li, J. Feng, and H.-B. Sun, “Optical Tamm states enhanced broad-band absorption of organic solar cells", Appl. Phys. Lett.101, 243901 (2012). 
17. X. L. Zhang, J. Feng, X. C. Han, Y. F. Liu, Q. D. Chen, J. F. Song, and H. B. Sun, "Hybrid Tamm plasmon-polariton/microcavity modes for white top-emitting organic light-emitting devices," Optica 2, 579584 (2015).

18. J. L. Kwang, J. W. Wu, and K. Kim, "Enhanced nonlinear optical effects due to the excitation of optical Tamm plasmon polaritons in one-dimensional photonic crystal structures," Opt. Express 21, 28817-28823 (2013).

19. V. A. Belyakov, Diffraction Optics of Complex Structured Periodic Media, (Springer Verlag, New York, 1992).

20. L. M. Blinov, Structure and Properties of Liquid Crystals (Springer, 2011), Chap. 12.

21. Y. C. Hsiao, C. T. Hou, V. Y. Zyryanov, and W. Lee, "Multichannel photonic devices based on tristable polymer-stabilized cholesteric textures," Opt. exp., 19, 23952-23957 (2011).

22.J.-C. Huang, Y.-C. Hsiao, Y.-T. Lin, C.-R. Lee, and W. Lee, "Electrically switchable organo-inorganic hybrid for a white-light laser source," Sci. Rep. 6, 28363 (2016).

23. S. Ya. Vetrov, M. V. Pyatnov, and I. V. Timofeev, "Surface modes in "photonic cholesteric liquid crystal-phase plate-metal" structure," Opt. Lett. 39, 2743-2746 (2014).

24. S. Ya. Vetrov, M. V. Pyatnov, and I. V. Timofeev, "Spectral and polarization properties of a 'cholesteric liquid crystal-phase platemetal'structure," J. Opt. 18, 015103 (2015).

25. M. V. Pyatnov, S. Ya. Vetrov, and I. V. Timofeev, "Localised optical states in a structure formed by two oppositely handed cholesteric liquid crystal layers and a metal," Liq. Cryst. 44, 674-678 (2017).

26. I.V. Timofeev, S.Y. Vetrov, "Chiral optical Tamm states at the boundary of the medium with helical symmetry of the dielectric tensor," JETP Lett. 104, 380-383 (2016).

27. I. V. Timofeev, P. S. Pankin, S. Y. Vetrov, V. G. Arkhipkin, W. Lee, and V. Y. Zyryanov, "Chiral Optical Tamm States: Temporal Coupled-Mode Theory," Crystals 7, 113 (2017).

28. A. H. Gevorgyan, and M. Z. Harutyunyan "Chiral photonic crystals with an anisotropic defect layer," Phys. Rev. E 76, 031701 (2007).

29. Y. C. Hsiao, H. T. Wang, and W. Lee, "Thermodielectric generation of defect modes in a photonic liquid crystal," Optics exp. 22, 35933599 (2014).

30. M. H. Song, B. Park, K. C. Shin, T. Ohta, Y. Tsunoda, H. Hoshi, Y. Takanishi, K. Ishikawa, J. Watanabe, S. Nishimura, T. Toyooka , Z. Zhu, T.M. Swager, and H. Takezoe, "Effect of Phase Retardation on Defect-Mode Lasing in Polymeric Cholesteric Liquid Crystals," Adv. Mat. 16, 779-783 (2004).

31. M. Mitov, "Cholesteric liquid crystals with a broad light reflection band," Adv. Mat. 24, 6260-6276 (2012).

32. R. B. Alaverdyan, K. R. Allakhverdyan, A. H. Gevorgyan, A. D.Chilingaryan, Y. S. Chilingaryan, "Chiral photonic crystals with an electrically tunable anisotropic defect. Experiment and theory," Tech. Phys. 55, 1317-1323 (2010).

33. Y. Matsuhisa, R. Ozaki, K. Yoshino, and M. Ozaki, "High Q defect mode and laser action in one-dimensional hybrid photonic crystal containing cholesteric liquid crystal," Appl. Phys. Lett, 89, 101109 (2006).

34. P. B. Johnson and R. W. Christy, "Optical constants of the noble metals,"Phys. Rev. B 6, 4370-4379 (1972).

35. D. W. Berreman, "Optics in Stratified and Anisotropic Media: 4 X 4Matrix Formulation", J. Opt. Soc. Am. 62, 502-510 (1972).

36. Y. C. Yang, C. S. Kee, J. E. Kim, H. Y. Park, J. C. Lee, and Y. J. Jeon, "Photonic defect modes of cholesteric liquid crystals," Phys. Rev. E 60, 6852. (1999).

37. V. A. Belyakov, and S. V. Semenov, "Optical edge modes in photonic liquid crystals." J. Exp. Theor. Phys. 109, 687-699 (2009).

38. A. L. Rodarte, F. Cisneros, L. S. Hirst, and S. Ghosh, "Dye-integrated cholesteric photonic luminescent solar concentrator." Liq. Cryst. 41, 1442-1447 (2014). 